Article

\title{
The Effect of Three Different Strategies Based on Motor Task Performance on Neuromuscular Fatigue in Healthy Men and Men with Multiple Sclerosis
}

\author{
Laura Kyguolienè ${ }^{1, *}$, Albertas Skurvydas ${ }^{1,2}$, Nerijus Eimantas ${ }^{2}$, Neringa Baranauskiené ${ }^{2}$, \\ Renata Balnytè ${ }^{3}$ (D) and Marius Brazaitis ${ }^{1,2}$ \\ 1 Department of Applied Biology and Rehabilitation, Lithuanian Sports University, Kaunas 44221, Lithuania; \\ albertas.skurvydas@1su.lt (A.S.); marius.brazaitis@lsu.lt (M.B.) \\ 2 Sports Science and Innovation Institute, Lithuanian Sports University, Kaunas 44221, Lithuania; \\ nerijus.eimantas@lsu.lt (N.E.); neringa.baranauskiene@lsu.lt (N.B.) \\ 3 Department of Neurology, Medical Academy, Lithuanian University of Health Science, \\ Kaunas 44307, Lithuania; renatabalnyte@gmail.com \\ * Correspondence: lauravalonyte@yahoo.com; Tel.: +370-610-180-80
}

Received: 18 March 2018; Accepted: 11 May 2018; Published: 24 May 2018

\begin{abstract}
Background and objectives: Fatigue during physical activity occurs because of decreased neuromuscular function. The aim of this study was to evaluate the effect of three different strategies based on motor task performance on neuromuscular fatigue in healthy men and men with multiple sclerosis (MS). Materials and Methods: We studied age-matched (18-43 years of age) healthy men $(n=15)$ and men with MS $(n=9)$. The inclusion criteria for MS subjects were a Kurtzke Expanded Disability Status Score $<4$ and a Fatigue Severity Scale Score $>5$. Both groups performed one of three exercise trials (with at least a 1-week interval between them) of 100 intermittent isometric knee extensions with flexion of $60^{\circ}$. The three different experimental conditions (ECs) were intermittent isometric contraction tasks with constant, predictable, and unpredictable torque target sequences. The variation of maximal voluntary contraction contractions (MVCs) within the strategies was $25 \%$, $50 \%$, and $75 \%$, with a set average of $50 \%$. All of them had a $5 \mathrm{~s}$ contraction and a $20 \mathrm{~s}$ rest period. The variables were measured: before exercise, after 100 repetitions (100-Reps), and $1 \mathrm{~h}$ after exercise. Results: In all EC tasks, the central activation ratio values of healthy and MS subjects were significantly different; however, no significant differences were observed among the EC tasks. No significant differences were seen in electrically induced torque, MVC torque, muscle temperature, subjective sensation of effort, coefficient of variation, or constant and absolute error after 100-Reps and $1 \mathrm{~h}$ after exercise between the two groups and in all EC tasks. Conclusions: Men with MS experienced higher central motor fatigue than did healthy men, but this had no effect on the variability, accuracy, or force sensation of the movements performed.
\end{abstract}

Keywords: multiple sclerosis; motor task specificity; motor accuracy; subjective force sensations

\section{Introduction}

Fatigue occurs because of exercise-induced limitations in skeletal muscles or in the nervous system [1-5]. The terms "central fatigue" and "peripheral fatigue" have been used to differentiate these two possible places of muscle fatigue [1,3]. Fatigue is one of the most common symptoms of multiple sclerosis (MS) and can have a major effect on health-related quality of life [6,7]. However, no universal agreement has been reached concerning the formal definition of fatigue, and its causes and mechanisms are not understood. 
It has been reported that persons with MS experience increased motor fatigue compared with healthy control persons during constant, repetitive contractions and ambulation [3,8,9]. Strong evidence suggests that the fatigue associated with MS results from reduced voluntary activation of muscles via central mechanisms $[3,8,10]$.

Exercise can be a helpful rehabilitation approach for individuals with MS to reestablish function, endorse wellness, and increase involvement in activities of daily living [11-13]. Moreover, accumulating evidence suggests that persons with MS are less physically active than are non-diseased populations [12].

Evidence exists for endorsing involvement in endurance training at low-to-moderate intensity, as the existing literature reveals that persons with MS can both tolerate and benefit from this training modality [14]. In addition, moderate-intensity resistance training also seems to be well tolerated and to have beneficial effects on patients with MS [15,16].

Recently, we found that intermittent isometric contractions (IICs) increased central and peripheral fatigue, force sensation, and intramuscular temperature and diminished absolute and constant error with no visual feedback (VF), but had no effect on motor variability [17] in healthy persons. Insignificant differences were found between the IIC (predictable vs. unpredictable) task strategies.

A literature review revealed an absence of previous studies of central and peripheral motor fatigue in persons with MS based on IIC strategies using constant, predictable, and unpredictable motor tasks. We hypothesized that the nervous systems of these patients would be more fatigable than those of healthy men during the performance of exercises; thus, patients with MS would tend to experience: (a) higher central motor fatigue by performing IIC tasks with submaximal intensity, which would lead to an increase in motor variability and a higher number of errors; and (b) a higher central motor fatigue and motor variability by performing IIC tasks using an unpredictable strategy vs. performing IIC tasks using constant and predictable strategies.

\section{Materials and Methods}

\subsection{Subjects}

Two groups of subjects were recruited: 15 healthy young men (age range, 19-27 years) and nine male patients with MS (age range, 21-43 years; disease duration, $7.1 \pm 3.0$ years). All subjects had normal and/or corrected vision. Written informed consent was obtained from all participants after receiving clarification of all details of the investigational actions and the related inconveniences. All procedures were approved by the Human Research Ethics Committee and were conducted according to the guidelines of the Declaration of Helsinki. The Kaunas Regional Ethics Committee approved this study (No. BE-2-35). The criteria for inclusion in the MS group were a Kurtzke Expanded Disability Status Score (EDSS) $<4$ [18] and a Fatigue Severity Scale (FSS) Score $>5$ [19]. Fatigue was quantified using the MS-specific questionnaire (FSS), which contains nine items rated on a 7-point Likert scale. Disease in the MS group was of the relapsing-remitting type, according to the McDonald criteria [20].

All volunteers participated in recreational activities two or three times per week and were considered physically active, without taking part in any recognized physical exercise or sports program. All volunteers had a leading right leg, as assessed by self-reports of their favored kicking leg. The participants were nonsmokers and had no history of significant knee injury or surgery, pain during resisted knee extension, pain, or instability during functional activities, or fracture of the pelvis, femur, tibia, fibula, or patella within the previous two years. We excluded patients who had other neurological or psychiatric malfunctions, such as depression or anxiety, due to their recognizable connection with fatigue [21]. Individuals using prednisone or antispasmodic drugs were also excluded. 


\subsection{Experimental Design}

A week prior the experimental trial, all subjects participated in an acquaintance session. Prior to the arrival at the laboratory, anthropometric variables were measured, and the experimental procedures for neuromuscular examination were demonstrated. All volunteers learned to achieve and maintain a maximal effort of the knee extensor for 3-4 s with a $250 \mathrm{~ms}$ test train (TT) of stimulation at $100 \mathrm{~Hz}$ (TT-100Hz) superimposed on a maximal voluntary contraction (MVC) to assess their tolerance to electrical stimulation (section "Isometric torque and electrical stimulation"). The volunteers also learned to maintain a force target at different torque intensities. They were tutored to refrain from consuming any food for a minimum of $12 \mathrm{~h}$, to refrain from consuming alcohol and caffeine and engaging in heavy workout for a minimum of $24 \mathrm{~h}$, and to sleep for a minimum of $8 \mathrm{~h}$ prior to each investigational session. To standardize the state of hydration and the sense of thirst, the subjects could take still water as desired up until $60 \mathrm{~min}$ prior to the testing. Examinations were performed at a laboratory temperature of $21^{\circ} \mathrm{C}$ and $50 \%$ relative humidity.

\subsection{Experimental Protocol}

The protocol involved three differently organized exercise task sessions, each of which was carried out randomly and separated by a rest period of a minimum of 7 days. The tasks were performed indoors starting at the same time of day (7 AM), to minimize body temperature. Prior to the arrival at the laboratory, the participants were asked to wear short-sleeved shirts, shorts, and socks, and to lie down in a horizontal position for $10 \mathrm{~min}$. After the relaxation period and before the exercises began, intramuscular temperature $\left(\mathrm{T}_{\mathrm{mu}}\right)$ was measured.

Prior to the start of each trial, the subjects performed a 10 min warm-up on an electrically braked cycle ergometer (Ergo-Fit, Pirmasens, Germany) with a pedaling frequency that ranged between 60 and $70 \mathrm{rpm}$. The subjects were seated in a dynamometer chair, and stimulating electrodes were positioned over the quadriceps femoris muscle of their right leg. Subsequently, the force-generating capacity of the quadriceps muscle was evaluated by applying $1 \mathrm{~s}$ trains of electrical stimuli at $20 \mathrm{~Hz}$ and $100 \mathrm{~Hz}$. Nearly $3 \mathrm{~s}$ were required to change the frequency of stimulation. After a $20 \mathrm{~s}$ break, two $5 \mathrm{~s}$ MVCs were assed with a $20 \mathrm{~s}$ rest period between them. At about $3 \mathrm{~s}$ of MVC, a $250 \mathrm{~ms}$ TT-100 Hz was superimposed on the voluntary contraction. These TT- $100 \mathrm{~Hz}$ contractions were used to determine the voluntary activation of the knee extensors. MVC force was defined as the maximum force that could be sustained for 2-3 s. If the relative difference between these two MVCs was within 10\%, no further trial was needed [22].

The higher of the two MVCs was used to compute the submaximal target torque. The percentage torque to be reached and sustained ("target torque") was indicated by a horizontal line on the screen of the dynamometer. We set the visual refreshing rate frequency at $2 \mathrm{kHz}$ on a standard monitor (Dell P2717H); the refresh rate was $60 \mathrm{~Hz}$, the resolution was full high definition $(1920 \times 1080$ pixels), the brightness/contrast ratio was 1:1, and a continuous line signal was provided. The participants then performed the IIC tasks under one of the three experimental conditions (ECs; constant, predictable, and unpredictable). All three IIC ECs included 100 isometric contractions, followed by a $1 \mathrm{~h}$ rest period and six IICs. The neuromuscular variables, i.e., electrical stimulus, MVC, central activation ratio (CAR), and $\mathrm{T}_{\mathrm{mu}}$, were measured three times in each session: before exercise, after 100 contractions (repetitions or Reps), and $1 \mathrm{~h}$ after exercise (Figure 1A). 
A

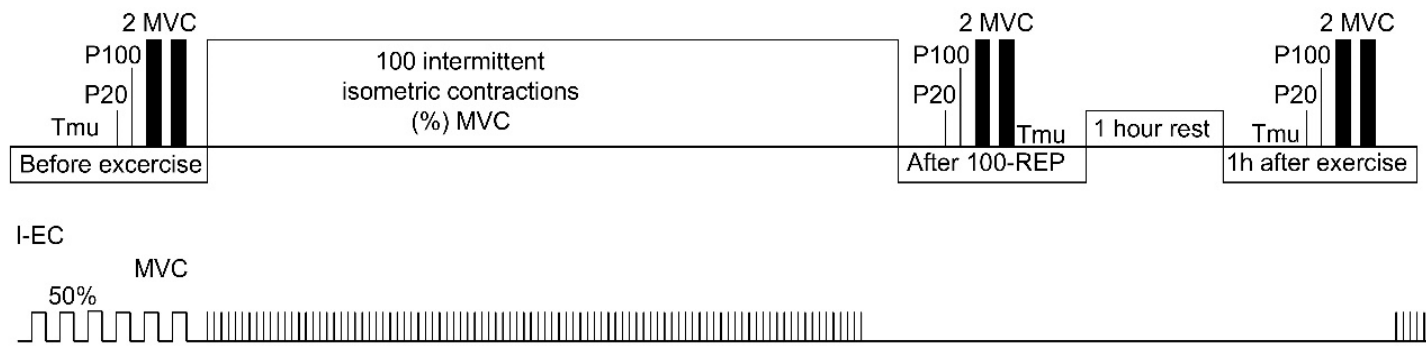

II-EC

لـ

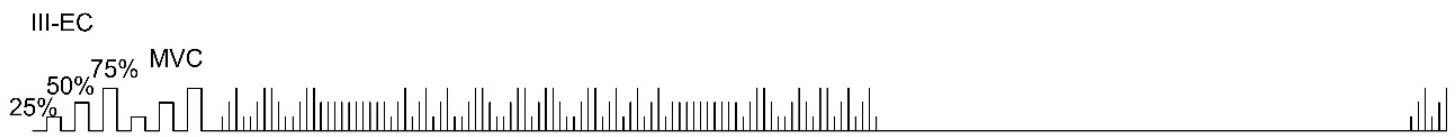

Figure 1. Experimental protocol. (A) Scheme of the experimental protocol; (B) Structural pattern illustration of the task for the three experimental conditions (ECs). Maximal voluntary contraction (MVC) torque, intramuscular temperature $\left(\mathrm{T}_{\mathrm{mu}}\right)$ and electrically induced quadriceps femoris muscle isometric torque caused by stimuli at $20 \mathrm{~Hz}$ (P20), $100 \mathrm{~Hz}$ (P100).

\subsection{The Three IIC ECS}

In each set of 100 Reps, knee extension was sustained for $5 \mathrm{~s}$, and the muscle was then allowed to relax for $20 \mathrm{~s}$. The torque output sum of 100 Reps under the three different IIC strategies was the same and corresponded to a mean intensity of $50 \%$ MVC. The main difference between the IIC tasks was the combination of muscle contraction torque intensity positions. Evidence indicates that the repeated performance of intermittent contractions during constant or predictable (i.e., stable) torque target sequence tasks elicits a learning effect. In the context of this monotonically adapted or learned motor task, unexpected (unpredictable) changes in the torque level in a selected task segment produce an unstable motor behavior, and greater cognitive-motor resources are required to perform the correct task-directed effort [23]. In consideration of this, the first EC (I-EC) comprised a constant torque target sequence at $50 \%$ of MVC; the second EC (II-EC) comprised an IIC task with a predictable torque target sequence at $25 \%, 50 \%$, or $75 \%$ of MVC; and the third EC (III-EC) involved an unpredictable torque target sequence at $25 \%, 50 \%$, or $75 \%$ of MVC. After each of the EC tasks, six other IICs were performed to provide an indication of muscle contraction steadiness after resting (Figure 1B).

Prior to and throughout each trial, the subjects were not introduced to the structure or sequence of the ECs and were blindfolded to block their view of the video display screen. The blindfold was then removed, and the participant had to perform the IIC to reach the upper torque line seen on the video display screen as quickly as possible and sustain the position for $5 \mathrm{~s}$ as smoothly and precisely as possible. Following the attempt, the covering was placed over their eyes and the participant rested for $20 \mathrm{~s}$ before the subsequent attempt. Throughout resting, the torque line was changed with reference to the EC protocol sequence, and the participant had no knowledge of where the torque line would appear on the next attempt.

\subsection{Measurements}

Throughout the initial visit, the height $(\mathrm{cm})$ of subjects was measured, and their weight $(\mathrm{kg})$, body mass index $\left(\mathrm{kg} / \mathrm{m}^{2}\right)$, fat-free mass $(\mathrm{kg})$, and body fat $(\%)$ were estimated by taking their nude body mass using a body composition analyzer (TBF-300; Tanita, UK Ltd., West Drayton, UK) (Table 1). 


\subsection{Isometric Torque and Electrical Stimulation}

The isometric torque of knee extensor muscles was measured using an isokinetic dynamometer (System 3; Biodex Medical Systems, Shirley, NY, USA), and data were acquired using dedicated software (Version 2.15; Biodex Medical Systems, Shirley, NY, USA). The subjects sat upright in the dynamometer chair with their knees positioned at a $60^{\circ}$ flexion ( $0^{\circ}$ full extension). Their shank, trunk, and shoulder were stabilized by belts. VF of torque from the dynamometer was provided on a video display screen located in front of the participants during the trial. The screen was positioned at eye level about $1 \mathrm{~m}$ in front of the participants. Electrical stimulation was applied directly to the muscles using two carbonized rubber electrodes covered with a thin layer of electrode gel (ECG-EEG Gel; Medigel, Modi'in, Israel). One of the electrodes $(6 \mathrm{~cm} \times 11 \mathrm{~cm})$ was placed transversally across the width of the proximal portion of the quadriceps femoris. Another electrode $(6 \mathrm{~cm} \times 20 \mathrm{~cm})$ covered the distal portion of the muscle above the patella. Stimulation was applied using a standard electrical stimulator (MG 440; Medicor, Budapest, Hungary) and was delivered in 0.5 ms square-wave pulses. Peak torques induced by $1 \mathrm{~s}$ of electrical stimulation at $20 \mathrm{~Hz}$ (P20; representing the steep section of the force-frequency relationship curve) and $100 \mathrm{~Hz}$ (P100; which is close to the maximal force) were measured with a 3 s rest interval between electrical stimulations [22,24]. The change in the P20 to P100 ratio (P20/P100) was used as a proxy of low-frequency fatigue [25]. The tolerance to electrical stimulation was evaluated during the introduction session, and only participants who exhibited good compliance with the procedure were employed in the study.

The intensity of the electrical stimulation was selected individually by applying single stimuli to the quadriceps muscle. The voltage was increased gradually, in $3 \mathrm{~V}$ increments. The voltage at which the participant first felt pain was defined as the pain threshold, and the voltage that could no longer be tolerated by the participant was defined as the pain tolerance [17]. During this procedure, the voltage was increased until no increment in single twitch torque could be detected with an additional $10 \%$ increase in the voltage.

After the tolerance to the electrical stimulation was evaluated at rest, two to three brief MVCs were required from subjects without superimposed TT-100Hz; subsequently, the same two to three brief MVCs, with a supplementary test trial (TT-100Hz) superimposed on an MVC, were terminated. Thus, if the relative difference between MVCs without superimposed TT- $100 \mathrm{~Hz}$ and MVCs with superimposed TT- $100 \mathrm{~Hz}$ was within $10 \%$, the tolerance to TT- $100 \mathrm{~Hz}$ during brief MVCs was indicated as acceptable [22].

\subsection{Central Activation Ratio (CAR) Measurements}

The CAR is the ratio of the maximal voluntary torque to the peak torque generated with a supplementary TT-100 Hz superimposed on an MVC. To evaluate the voluntary activation of knee extensors, a $250 \mathrm{~ms}$ trial of stimuli at $100 \mathrm{~Hz}$ (TT-100 Hz) was superimposed on the voluntary contraction at about $3 \mathrm{~s}$ of MVC. The CAR was computed as defined elsewhere $[2,22,26]$ using the following equation:

$$
\mathrm{CAR}=\mathrm{MVC} /(\mathrm{MVC}+\mathrm{TT}-100 \mathrm{~Hz}) \times 100 \%
$$

where a CAR of $100 \%$ indicates complete activation of the exercising muscle and a CAR $<100 \%$ indicates central activation failure or inhibition.

\subsection{Muscle Temperature Measurements}

$\mathrm{T}_{\mathrm{mu}}$ was evaluated using a needle microprobe (MKA; Ellab, Hvidovre, Denmark) inserted $3 \mathrm{~cm}$ under the skin and covering the vastus lateralis muscle of the right leg [27]. Skin preparation prior to each $\mathrm{T}_{\mathrm{mu}}$ measurement involved skin shaving and disinfection with a cotton-wool tampon soaked in isopropyl alcohol before and after insertion. Insertion was made without any anesthesia. After the first measurement, the insertion area was marked with a circle with a diameter of $0.5 \mathrm{~cm}$. 


\subsection{Rating of Perceived Exertion (RPE)}

The subjects were requested to rate their perceived exertion on the Borg scale, with values ranging from 6 (no exertion at all) to 20 (maximal exertion). The RPE was measured with VF at $50 \%$ of the required torque at the time points (before exercise, after 100 Reps, and $1 \mathrm{~h}$ after exercise).

\subsection{Accuracy and Motor Variability of IIC Assessment}

The accuracy of the $5 \mathrm{~s}$ target IIC torque at $50 \%$ of MVC was computed as the constant error and absolute error; however, the coefficient of variation was computed to estimate the intraindividual motor variability of $5 \mathrm{~s}$ IICs. Attention was paid to algebraic symbols $( \pm)$. The absolute error specifies the absolute deviation from the required target force ( $50 \%$ of the isometric force required).

$$
\text { Constant error }=\sum\left(x_{i}-T\right) / n
$$

where $x_{i}$ is the IIC performed (N.m); $T$ is the target quantity, i.e., the IIC required; $n$ is the number of trials; and $\Sigma$ indicates the mean that was calculated considering the algebraic symbols $( \pm)$.

$$
\text { Absolute error }=\sum\left|x_{i}-T\right| / n
$$

where $x_{i}$ is the IIC performed $(\mathrm{N} \cdot \mathrm{m}) ; T$ is the target quantity, i.e., the IIC required; $n$ is the number of trials; and vertical brackets $\Sigma||$ indicate the mean that was calculated without considering the algebraic symbols $( \pm)$.

$$
\text { Coefficient of variantion (normalized variable error })=(\mathrm{SD} / \bar{x}) \times 100
$$

where SD is the mean standard deviation of IICs performed; $\bar{x}$ and is the mean IIC $(\mathrm{N} \cdot \mathrm{m})$.

\subsection{Statistical Analyses}

The normality of the data distribution was evaluated using the Kolmogorov-Smirnov test; all data were found to be normally distributed, except the Borg scale. Single-time-point comparisons between groups were analyzed using independent sample $t$-tests for the baseline values (physical characteristics).

Repeated-measures analysis of variance (ANOVA) was used to study the effects of the three ECs (I-EC vs. II-EC vs. III-EC) as a within-subject factor for $\mathrm{T}_{\mathrm{mu}}$, the accuracy and variability of IIC, CAR, $\mathrm{MVC}$, and the electrically induced torque before exercise.

A mixed ANOVA was performed with one between-subjects factor (groups: healthy men vs. MS men) and multiple within-subject factors (I-EC vs. II-EC vs. III-EC) and time (before exercise vs. 100 Reps vs. $1 \mathrm{~h}$ after exercise) for electrically induced torque, $\mathrm{T}_{\mathrm{mu}}, \mathrm{MVC}$ and CAR, the coefficient of variation, and constant and absolute errors (i.e., the accuracy and variability of IIC).

If significant effects were found, Sidak's post-hoc adjustment was used for multiple comparisons within each repeated-measure ANOVA. Calculations of statistical power (SP, as a percentage) were performed for all indicators based on an alpha level of 0.05 , sample size $(n=11)$, standard deviations, and changes in the average level of the data. The SP for a significant effect was $>80 \%$. The partial eta squared $\left(\eta_{p}{ }^{2}\right)$ was estimated as a measure of the EC effect size. The nonparametric Wilcoxon signed rank test for two related samples was used to compare changes in subjective ratings of effort sensation. Descriptive data are presented as the mean \pm standard deviation. The significance of all tests was set at $p<0.05$. Data were analyzed using SPSS version 21.0 (IBM Corp., Armonk, NY, USA). 


\section{Results}

\subsection{Participants' Characteristics}

Table 1 describes the reference characteristics of the participants. Healthy men and men with MS had similar height, mass, BMI, fat-free mass, body fat \%, and age, with significant differences between groups $(p<0.05)($ Table 1$)$.

Table 1. Physical characteristics of the participants.

\begin{tabular}{lll}
\hline & Healthy Men $(n=15)$ & MS Men $(n=9)$ \\
\hline Age, yr. & $22.47 \pm 3.74$ & $31.11 \pm 8.51^{\#}$ \\
Height, cm & $182.53 \pm 6.77$ & $178.67 \pm 5.79$ \\
Mass, kg & $78.51 \pm 8.09$ & $73.13 \pm 6.72$ \\
Body mass index, $\mathrm{kg} / \mathrm{m}^{2}$ & $23.58 \pm 2.29$ & $22.90 \pm 1.53$ \\
Fat free mass, $\mathrm{kg}$ & $12.67 \pm 4.34$ & $12.42 \pm 3.99$ \\
Body fat, \% & $15.86 \pm 4.15$ & $16.77 \pm 4.46$ \\
EDSS, points & & $3.1 \pm 1.3$ \\
\hline
\end{tabular}

Expanded Disability Status Score (EDSS). Values are means \pm standard deviation. ${ }^{\#} p<0.05$ when comparing healthy men and men with multiple sclerosis.

\subsection{Baseline Neuromuscular and Intramuscular Temperature $\left(T_{m u}\right)$ Characteristics}

Table 2 presents the baseline data of MVC torque, CAR, and $\mathrm{T}_{\mathrm{mu}}$. Generally, men with MS were weaker than healthy men. Table 2 shows that, for the initial values obtained in all EC tasks, men with MS had lower MVC torque, CAR, and $\mathrm{T}_{\mathrm{mu}}$ compared with healthy men $\left(p<0.001, \eta_{p}{ }^{2}=0.54, \mathrm{SP}>99 \%\right.$, $p<0.05, \eta_{p}{ }^{2}=0.22, \mathrm{SP}>80 \%$, and $p<0.05, \eta_{p}{ }^{2}=0.33, \mathrm{SP}>85 \%$, respectively). No differences were observed between the initial values of men with MS and healthy men in before-exercise MVC torque, CAR, or $\mathrm{T}_{\mathrm{mu}}$ among all EC tasks $(p>0.05)$ in (Table 2$)$.

Table 2. Reference characteristics of maximal voluntary contraction (MVC) torque, central activation ratio $(C A R)$, and intramuscular temperature $\left(\mathrm{T}_{\mathrm{mu}}\right)$ in healthy men and men with multiple sclerosis (MS) before exercise in 3 experimental conditions (ECs).

\begin{tabular}{lllll}
\hline & & MVC $(\mathbf{N} \cdot \mathbf{m})$ & CAR $(\%)$ & $\mathbf{T}_{\mathbf{m u}}\left({ }^{\circ} \mathbf{C}\right)$ \\
\hline \multirow{2}{*}{ I-EC } & Healthy men & $316.79 \pm 44.42$ & $96.22 \pm 3.65$ & $36.9 \pm 0.5$ \\
& MS men & $234.51 \pm 39.83^{\#}$ & $92.44 \pm 4.722^{\#}$ & $36.2 \pm 0.5^{\#}$ \\
\hline \multirow{2}{*}{ II-EC } & Healthy men & $310.83 \pm 40.16$ & $95.15 \pm 5.25$ & $36.9 \pm 0.4$ \\
& MS men & $228.84 \pm 41.37^{\#}$ & $90.55 \pm 6.87^{\#}$ & $36.0 \pm 1.0^{\#}$ \\
\hline \multirow{2}{*}{ III-EC } & Healthy men & $320.28 \pm 36.40$ & $96.57 \pm 2.83$ & $36.7 \pm 0.6$ \\
& MS men & $231.57 \pm 47.88^{\#}$ & $92.55 \pm 5.22^{\#}$ & $36.3 \pm 0.6^{\#}$ \\
\hline
\end{tabular}

Values are means \pm standard deviation. ${ }^{*} p<0.05$ when comparing healthy men and men with MS.

\subsection{Maximal Voluntary Contraction (MVC) Torque, Intramuscular Temperature $\left(T_{m u}\right)$, Central Activation Ratio (CAR) and Rating of Perceived Exertion (RPE)}

In all EC tasks, MVC torque diminished after 100 Reps and remained unrecovered $1 \mathrm{~h}$ after exercise compared with the values recorded before exercise $\left(p<0.001, \eta_{p}{ }^{2}=0.8\right.$, SP $>95 \%$; and $p<0.05$, $\eta_{p}{ }^{2}=0.6, \mathrm{SP}>85 \%$, respectively) for both healthy controls and men with MS (Figure 2A).

Figure 2B shows that the $\mathrm{T}_{\mathrm{mu}}$ for both healthy controls and men with MS increased after 100 Reps $\left(p<0.001, \eta_{p}{ }^{2}=0.98, \mathrm{SP}>99 \%\right.$; and $p<0.001, \eta_{p}{ }^{2}=0.85, \mathrm{SP}>99 \%$, respectively $)$ and returned to before-exercise values after $1 \mathrm{~h}$ of recovery $(p>0.05)$ in all EC tasks.

In all EC tasks, the CAR of healthy men decreased after 100 Reps $\left(p<0.05, \eta_{p}^{2}=0.75, \mathrm{SP}>80 \%\right)$ and returned to before-exercise values after $1 \mathrm{~h}$ of recovery. The CAR of men with MS decreased after 
100 Reps and remained unrecovered to pre-exercise values within $1 \mathrm{~h}$ after the exercise in the I-EC and III-EC trials $\left(p<0.05, \eta_{p}{ }^{2}=0.4, \mathrm{SP}>80 \% ; p<0.05, \eta_{p}{ }^{2}=0.5\right.$, SP $>85 \%$, respectively) (Figure $2 \mathrm{C}$ ). Conversely, the II-EC trial resulted in no differences in the CAR values of the 100 Reps and $1 \mathrm{~h}$ after exercise conditions compared with the values recorded before exercise. In all EC tasks, the CAR values of healthy and MS subjects were insignificantly different between groups $\left(p<0.05, \eta_{p}{ }^{2}=0.33\right.$, SP $\left.>85 \%\right)$ (Figure 2C).

In all EC tasks, the subjective sensation of effort for both healthy and MS subjects increased after 100 Reps and remained constant at $1 \mathrm{~h}$ after exercise $\left(p<0.001, \eta_{p}{ }^{2}=0.9, \mathrm{SP}>99 \% ; p<0.05, \eta_{p}{ }^{2}=0.6\right.$, SP $>95 \%$, respectively) (Figure 2D).

However, a comparison of MVC torque, $\mathrm{T}_{\mathrm{mu}}$, and the subjective sensation of effort in all EC tasks resulted in nonsignificant differences between the two groups. Figure 2 shows an absence of significant differences in MVC, $\mathrm{T}_{\mathrm{mu}}, \mathrm{CAR}$, and the subjective sensation of effort among the EC tasks $(p>0.05)$.
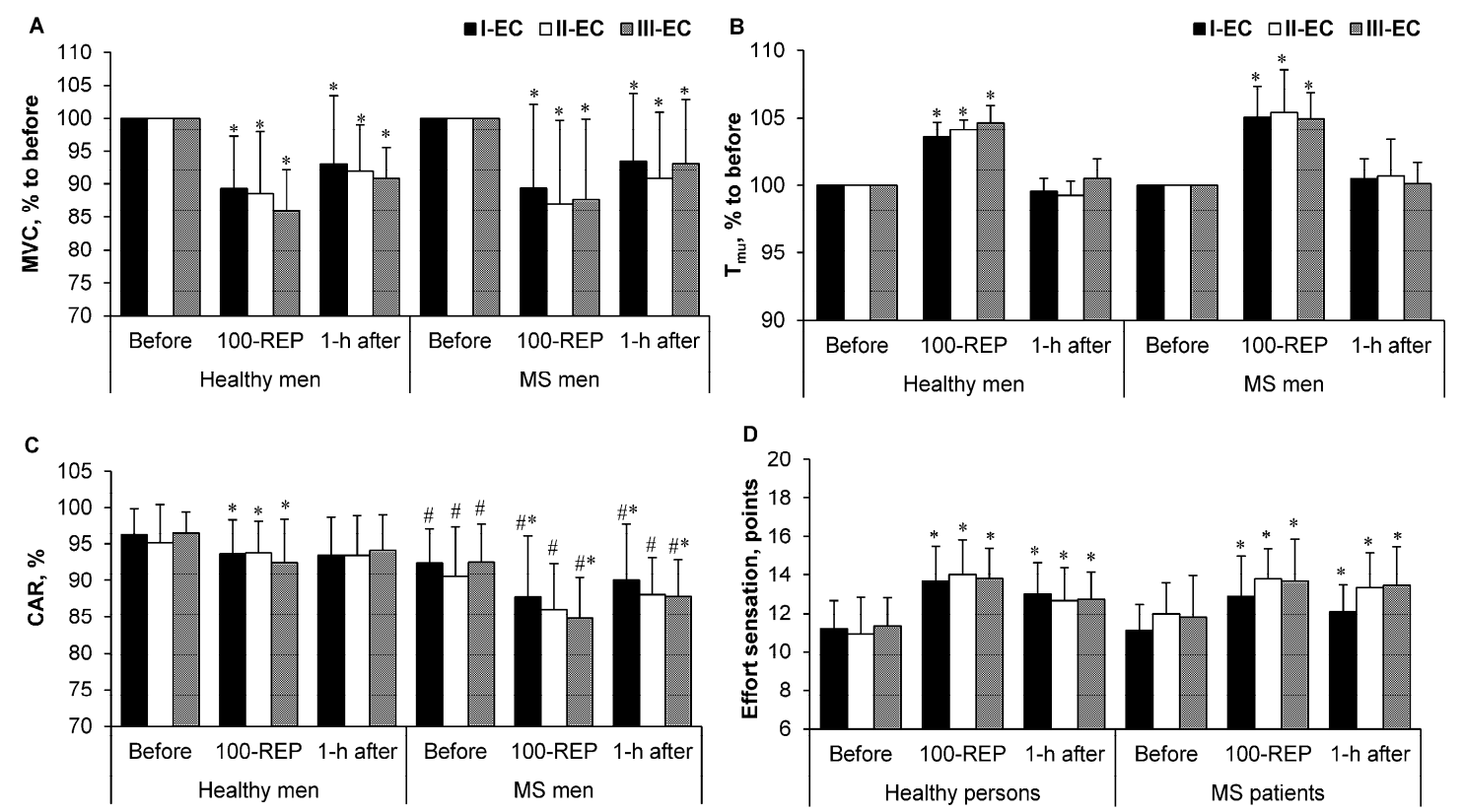

Figure 2. (A) Maximal voluntary contraction (MVC) torque and (B) intramuscular temperature $\left(\mathrm{T}_{\mathrm{mu}}\right)$ after 100 repetitions (100-REP) and at $1 \mathrm{~h}$ after exercise in the three experimental conditions (ECs). Values of MVC torque and $\mathrm{T}_{\mathrm{mu}}<100 \%$ were taken from the initial measurements in these experiments. (C) Central activation ratio (CAR) and (D) effort sensation values before, after 100 repetitions (100-REP), and at $1 \mathrm{~h}$ after exercise in the three ECs. Values are expressed as means \pm standard deviation. ${ }^{*} p<0.05$ compared with "before exercise"; $" p<0.05$ when comparing healthy men and men with multiple sclerosis (MS).

\subsection{Electrically Induced Muscle Torque}

For all EC tasks, the electrically induced (P20) torque of healthy men decreased significantly after 100 Reps $\left(p<0.001, \eta_{p}^{2}=0.62, \mathrm{SP}>99 \%\right)$ and had recovered to before-exercise values at $1 \mathrm{~h}$ after exercise ( $p>0.05$ ) (Figure 3A). In all EC tasks, in the MS group, a comparison of the (P20) torque values of 100 Reps and at $1 \mathrm{~h}$ after exercise with the initial ones recorded before exercise revealed an absence of differences (Figure 3A).

In the group of healthy men (P100), the torque decreased significantly after 100 Reps, without regression to the before-exercise level at $1 \mathrm{~h}$ after exercise, for all EC tasks $\left(p<0.001, \eta_{p}{ }^{2}=\right.$ $0.68, \mathrm{SP}>99 \%$ ) (Figure 3B). In the MS group (P100), the torque decreased significantly after 100 Reps $\left(p<0.05 \eta_{p}{ }^{2}=0.47 \mathrm{SP}>85 \%\right)$, with regression to the before-exercise level within $1 \mathrm{~h}$ after the exercise $(p>0.05)$, for all EC tasks (Figure 3B). 
In both groups, the P20/P100 ratio increased at $1 \mathrm{~h}$ after exercise $\left(p<0.001, \eta_{p}{ }^{2}=0.6 \mathrm{SP}>99 \%\right.$; $p<0.05, \eta_{p}{ }^{2}=0.6 \mathrm{SP}>90 \%$, respectively) for all EC tasks (Figure 3C).

However, a comparison of (P20) with (P100) and the ratio of P20/P100 torques in all EC tasks resulted in nonsignificant differences between the two groups (Figure $3 \mathrm{~A}-\mathrm{C}$ ). Figure 3 shows the absence of significant differences in electrically induced quadriceps femoris muscle isometric torque at (P20), (P100), and the ratio of P20/P100 torques among all EC tasks $(p>0.05)$.
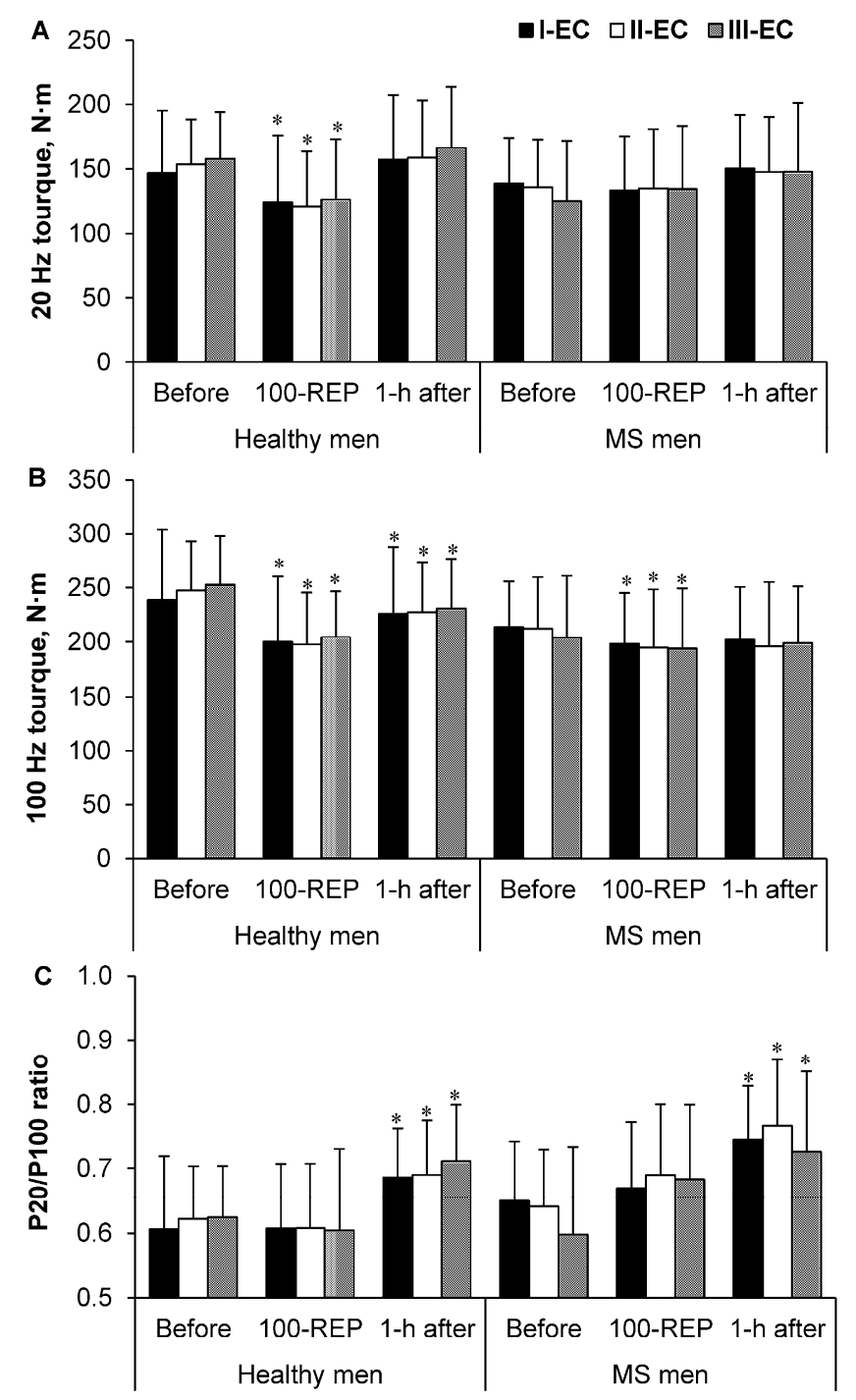

Figure 3. Electrically induced quadriceps femoris muscle isometric torque caused by stimuli at (A) $20 \mathrm{~Hz}$; (B) $100 \mathrm{~Hz}$; (C) the ratio of P20/P100 torques before exercise, 100 repetitions (100-REP), and at 1 $\mathrm{h}$ after exercise in the three experimental conditions (ECs). Values are expressed as means \pm standard deviation. * $p<0.05$ compared with "before exercise".

\subsection{Accuracy and Motor Variability of IIC Tasks}

Table 3 presents the data on the coefficient of variation, constant error, and absolute error values. The variation coefficient, constant, and absolute errors of 50\% isometric MVC did not differ significantly between the groups of healthy and MS subjects $(p>0.05)$. We found no effect of EC tasks on the variation coefficient, constant, or absolute errors (Table 3). 
Table 3. Coefficient of variation (CV), constant error (CE), and absolute error (AE) values before exercise, after 100 repetitions (100-REP), and $1 \mathrm{~h}$ after exercise in the three ECs.

\begin{tabular}{|c|c|c|c|c|}
\hline & & \multicolumn{3}{|c|}{ CV (\%) } \\
\hline & & Before Exercise & After 100-REP & 1-h after Exercise \\
\hline \multirow{2}{*}{ I-EC } & Healthy men & $4.1 \pm 1.7$ & $3.5 \pm 1.5$ & $3.3 \pm 1.5$ \\
\hline & MS men & $6.1 \pm 3.5$ & $3.0 \pm 1.9$ & $2.9 \pm 2.5$ \\
\hline \multirow{2}{*}{ II-EC } & Healthy men & $5.6 \pm 3.7$ & $5.0 \pm 2.1$ & $3.9 \pm 1.6$ \\
\hline & MS men & $5.1 \pm 3.6$ & $2.9 \pm 1.4$ & $4.8 \pm 4.8$ \\
\hline \multirow{3}{*}{ III-EC } & Healthy men & $6.8 \pm 8.3$ & $3.4 \pm 1.1$ & $3.4 \pm 2.0$ \\
\hline & MS men & $3.9 \pm 2.6$ & $3.3 \pm 1.5$ & $4.7 \pm 3.6$ \\
\hline & & \multicolumn{3}{|c|}{$\mathrm{CE}(\mathrm{N} \cdot \mathrm{m})$} \\
\hline \multirow{2}{*}{ I-EC } & Healthy men & $-4.4 \pm 4.9$ & $-2.1 \pm 6.4$ & $-2.6 \pm 5.9$ \\
\hline & MS men & $-5.21 \pm 4.01$ & $-5.07 \pm 5.17$ & $-6.39 \pm 5.86$ \\
\hline \multirow{2}{*}{ II-EC } & Healthy men & $-8.8 \pm 6.7$ & $-3.2 \pm 5.8$ & $-3.9 \pm 4.9$ \\
\hline & MS men & $-7.31 \pm 5.64$ & $-5.14 \pm 6.92$ & $-7.44 \pm 5.68$ \\
\hline \multirow{3}{*}{ III-EC } & Healthy men & $-7.4 \pm 8.9$ & $-3.5 \pm 5.2$ & $-6.6 \pm 9.9$ \\
\hline & MS men & $-6.87 \pm 5.09$ & $-4.23 \pm 4.11$ & $-7.63 \pm 4.27$ \\
\hline & & \multicolumn{3}{|c|}{$\mathrm{AE}(\mathrm{N} \cdot \mathrm{m})$} \\
\hline \multirow{2}{*}{ I-EC } & Healthy men & $7.1 \pm 2.6$ & $6.4 \pm 1.9$ & $6.4 \pm 2.9$ \\
\hline & MS men & $7.70 \pm 2.37$ & $6.89 \pm 2.10$ & $7.89 \pm 3.74$ \\
\hline \multirow{2}{*}{ II-EC } & Healthy men & $10.2 \pm 4.6$ & $7.0 \pm 2.1$ & $6.5 \pm 2.3$ \\
\hline & MS men & $7.61 \pm 5.42$ & $7.49 \pm 4.42$ & $7.69 \pm 5.39$ \\
\hline \multirow{2}{*}{ III-EC } & Healthy men & $9.3 \pm 8.1$ & $6.1 \pm 3.6$ & $7.4 \pm 9.6$ \\
\hline & MS men & $7.48 \pm 4.00$ & $5.88 \pm 1.94$ & $8.14 \pm 4.31$ \\
\hline
\end{tabular}

Data are shown as means \pm standard deviation.

\section{Discussion}

The main findings of our study are as follows:

(1) The decrease in MVC force was the same for both groups; however, the decrease observed in MS subjects occurred via central neural mechanisms, whereas in healthy men, it occurred through peripheral components;

(2) The CAR was lower for individuals with MS than for healthy men, although the effort sensation increased evenly and with no reference to IIC strategies;

(3) The central and peripheral motor fatigue of MS and healthy subjects had no reference to IIC strategies;

(4) No difference was observed in motor variability or movement accuracy in men with MS or healthy men in all three ECs considered. Neither the first nor second of our hypotheses were confirmed by the findings of our study. The first hypothesis was that men with MS would perform IIC with higher motor variability and a higher number of errors compared with healthy men. However, we found that, in all three considered ECs, the CAR of men with MS was lower than that of healthy men (Figure 2C). Moreover, it was assumed that a decrease in the CAR would lead to an increase in the coefficient of variation, and absolute and constant errors; however, the study yielded the opposite results, i.e., with any decrease of the CAR, no difference was seen in the coefficient of variation or absolute and constant errors in either men with MS or healthy men (Table 3). The absence of variation in these parameters could be indicative of the interaction between learning and fatigue. In the process of motor learning, the variability of movements decreases, and accuracy increases [28]; the increase in fatigue leads to the contrary tendency [2]. 
The second hypothesis assumed that throughout the performance of IIC tasks using the unpredictable strategy, the men with MS would experience higher central fatigue (a higher decrease of the CAR), which would increase the number of errors and motor variability. However, our results revealed that central motor fatigue, the number of errors, and variability were not correlated with IIC task strategy (Figure 2C). The learning of movements during the performance of IIC tasks could counterbalance the appearance of central fatigue [28]. Unfortunately, the study failed to distinguish the effects of each of those two processes.

Our findings reflect the results of previous research, indicating that during the performance of physical exercise, patients with MS experience higher central but lower peripheral fatigue [3,8-10]. Moreover, peripheral fatigue, i.e., decreases in electrically induced force, also depends on two processes: interactions of muscle potentiation, and fatigue [4,29]. Previous studies have shown that the performance of 100 repetitive maximal voluntary quadriceps muscle contractions leads to assertion of post-tetanic potentiation, low-frequency fatigue, and post-contractile depression [29].

Electrically induced isometric force production at $20 \mathrm{~Hz}$ (P20) did not exceed the value of $100 \mathrm{~Hz}$ (P100) force production (Figure 3A,B); thus, no evidence of low-frequency fatigue was seen. Increased muscle temperature evokes a decrease in involuntary muscle force [27]. In addition, during the time of recovery, an increase in the P20/P100 factor was observed in both groups, indicating a muscle potentiation mechanism before evidential fatigue [4]. This muscle potentiation mechanism may conceal an increase in fatigue-based muscle force sensation, i.e., a higher potentiation leads to a lower force sensation [30]. With respect to the predominance of chronic fatigue, men with MS [31] require potentiation of the motor system. Based on previous observations, the evidence of muscle potentiation during the performance of IIC tasks can be applicable as a form of rehabilitation, whereas peripheral fatigue does not differ between healthy and multiple sclerosis working-age adults, significantly affecting their daily efficiency.

\section{Conclusions}

The results of the present study revealed that men with MS exhibited higher central motor fatigue than did healthy men, although this had no effect on the variability, accuracy, or force sensation of the performed movements. This might be the result of muscle potentiation, motor learning, or motor fatigue. Consequently, because of motor fatigue potentiation on their muscles, men with MS could accomplish submaximal IIC, resulting in better task performance.

Author Contributions: The authors A.S. and M.B. contributed to the design of the work. The authors L.K., N.E., N.B., R.B., M.B. performed the experiments. The authors L.K., A.S. contributed to the analysis and interpretation of data for the work. The authors L.K., A.S. drafted the work for important intellectual content. The authors N.E., N.B., R.B., M.B. finally approved the version to be submitted. The author L.K. contributed to the revision of this work. All the authors agreed to be accountable for all aspects of the work in ensuring that questions related to the accuracy or integrity of any part of the work are appropriately investigated and resolved.

Funding: Funding for this work was provided by the Research Council of Lithuania, Grant no. MIP-017/2014.

Acknowledgments: The authors acknowledge the participants who volunteered for the study.

Conflicts of Interest: The authors declare no conflict of interest. The founding sponsors had no role in the design of the study; in the collection, analyses, or interpretation of data; in the writing of the manuscript, and in the decision to publish the results.

\section{References}

1. Gandevia, S.C. Spinal and supraspinal factors in human muscle fatigue. Physiol. Rev. 2001, 81, 1725-1789. [CrossRef] [PubMed]

2. Brazaitis, M.; Skurvydas, A.; Pukènas, K.; Daniuseviciūtè, L.; Mickevicienè, D.; Solianik, R. The effect of temperature on amount and structure of motor variability during 2-minute maximum voluntary contraction. Muscle Nerve 2012, 46, 799-809. [CrossRef] [PubMed] 
3. Skurvydas, A.; Brazaitis, M.; Andrejeva, J.; Mickeviciene, D.; Streckis, V. The effect of multiple sclerosis and gender on central and peripheral fatigue during 2-min MVC. Clin. Neurophysiol. 2011, 122, 767-776. [CrossRef] [PubMed]

4. Skurvydas, A.; Mamkus, G.; Kamandulis, S.; Dudoniene, V.; Valanciene, D.; Westerblad, H. Mechanisms of force depression caused by different types of physical exercise studied by direct electrical stimulation of human quadriceps muscle. Eur. J. Appl. Physiol. 2016, 116, 2215-2224. [CrossRef] [PubMed]

5. Taylor, J.L.; Amann, M.; Duchateau, J.; Meeusen, R.; Rice, C.L. Neural contributions to muscle fatigue: From the brain to the muscle and back again. Med. Sci. Sports Exerc. 2016, 48, 2294-2306. [CrossRef] [PubMed]

6. Johnson, S.L. The concept of fatigue in multiple sclerosis. J. Neurosci. Nurs. 2008, 40, 72-77. [CrossRef] [PubMed]

7. Leocani, L.; Colombo, B.; Comi, G. Physiopathology of fatigue in multiple sclerosis. Neurol. Sci. 2008, 29, S241-S243. [CrossRef] [PubMed]

8. Sheean, G.L.; Murray, N.M.; Rothwell, J.C.; Miller, D.H.; Thompson, A.J. An electrophysiological study of the mechanism of fatigue in multiple sclerosis. Brain 1997, 120 Pt 2, 299-315. [CrossRef] [PubMed]

9. Severijns, D.; Zijdewind, I.; Dalgas, U.; Lamers, I.; Lismont, C.; Feys, P. The assessment of motor fatigability in persons with multiple sclerosis: A systematic review. Neurorehabil. Neural Repair 2017, 31, 413-431. [CrossRef] [PubMed]

10. Kent-Braun, J.A.; Miller, R.G. Central fatigue during isometric exercise in amyotrophic lateral sclerosis. Muscle Nerve 2000, 23, 909-914. [CrossRef]

11. Dalgas, U. Exercise therapy in multiple sclerosis and its effects on function and the brain. Neurodegener. Dis. Manag. 2017, 7, 35-40. [CrossRef] [PubMed]

12. Motl, R.W.; Sandroff, B.M.; Kwakkel, G.; Dalgas, U.; Feinstein, A.; Heesen, C.; Feys, P.; Thompson, A.J. Exercise in patients with multiple sclerosis. Lancet Neurol. 2017, 16, 848-856. [CrossRef]

13. Zimmer, P.; Bloch, W.; Schenk, A.; Oberste, M.; Riedel, S.; Kool, J.; Langdon, D.; Dalgas, U.; Kesselring, J.; Bansi, J. High-intensity interval exercise improves cognitive performance and reduces matrix metalloproteinases- 2 serum levels in persons with multiple sclerosis: A randomized controlled trial. Mult. Scler. 2017, 1-10. [CrossRef] [PubMed]

14. Dalgas, U.; Stenager, E.; Ingemann-Hansen, T. Multiple sclerosis and physical exercise: Recommendations for the application of resistance-, endurance- and combined training. Mult. Scler. 2008, 14, 35-53. [CrossRef] [PubMed]

15. White, L.J.; Dressendorfer, R.H. Exercise and multiple sclerosis. Sports Med. 2004, 34, 1077-1100. [CrossRef] [PubMed]

16. Kjølhede, T.; Vissing, K.; de Place, L.; Pedersen, B.G.; Ringgaard, S.; Stenager, E.; Petersen, T.; Dalgas, U. Neuromuscular adaptations to long-term progressive resistance training translates to improved functional capacity for people with multiple sclerosis and is maintained at follow-up. Mult. Scler. 2015, 21, 599-611. [CrossRef] [PubMed]

17. Kyguoliene, L.; Skurvydas, A.; Eimantas, N.; Baranauskienè, N.; Mickeviciene, D.; Urboniene, D.; Cernych, M.; Brazaitis, M. Effect of constant, predictable, and unpredictable motor tasks on motor performance and blood markers of stress. Exp. Brain Res. 2017, 235, 1323-1336. [CrossRef] [PubMed]

18. Kurtzke, J.F. Rating neurologic impairment in multiple sclerosis: An expanded disability status scale. Neurology 1983, 33, 1444-1452. [CrossRef] [PubMed]

19. Krupp, L.B.; LaRocca, N.G.; Muir-Nash, J.; Steinberg, A.D. The fatigue severity scale. Application to patients with multiple sclerosis and systemic lupus erythematosus. Arch. Neurol. 1989, 46, 1121-1123. [CrossRef] [PubMed]

20. Thompson, A.J.; Banwell, B.L.; Barkhof, F.; Carroll, W.M.; Coetzee, T.; Comi, G.; Correale, J.; Fazekas, F.; Filippi, M.; Freedman, M.S.; et al. Diagnosis of multiple sclerosis: 2017 revisions of the McDonald criteria. Lancet Neurol. 2018, 17, 162-173. [CrossRef]

21. Simpson, S., Jr.; Tan, H.; Otahal, P.; Taylor, B.; Ponsonby, A.L.; Lucas, R.M.; Blizzard, L.; Valery, P.C.; Lechner-Scott, J.; Shaw, C.; et al. Anxiety, depression and fatigue at 5-year review following CNS demyelination. Acta Neurol. Scand. 2016, 134, 403-413. [CrossRef] [PubMed]

22. Bernecke, V.; Pukenas, K.; Imbrasiene, D.; Mickeviciene, D.; Baranauskiene, N.; Eimantas, N.; Brazaitis, M. Test-retest cross-reliability of tests to assess neuromuscular function as a multidimensional concept. J. Strength Cond. Res. 2015, 29, 1972-1984. [CrossRef] [PubMed] 
23. Osu, R.; Burdet, E.; Franklin, D.W.; Milner, T.E.; Kawato, M. Different mechanisms involved in adaptation to stable and unstable dynamics. J. Neurophysiol. 2003, 90, 3255-3269. [CrossRef] [PubMed]

24. Brazaitis, M.; Eimantas, N.; Daniuseviciute, L.; Vitkauskiene, A.; Paulauskas, H.; Skurvydas, A. Two strategies for the acute response to cold exposure but one strategy for the response to heat stress. Int. J. Hyperth. 2015, 31, 325-335. [CrossRef] [PubMed]

25. Venckunas, T.; Skurvydas, A.; Brazaitis, M.; Kamandulis, S.; Snieckus, A.; Moran, C.N. Human alpha actinin-3 genotype association with exercise-induced muscle damage and the repeated-bout effect. Appl. Physiol. Nutr. Metab. 2012, 37, 1038-1046. [CrossRef] [PubMed]

26. Kent-Braun, J.A. Central and peripheral contributions to muscle fatigue in humans during sustained maximal effort. Eur. J. Appl. Physiol. Occup. Physiol. 1999, 80, 57-63. [CrossRef] [PubMed]

27. Brazaitis, M.; Skurvydas, A.; Vadopalas, K.; Daniusevičiūtè, L.; Senikienè, Z. The effect of heating and cooling on time course of voluntary and electrically induced muscle force variation. Medicina (Kaunas) 2011, 47, 39-45. [CrossRef] [PubMed]

28. Müller, H.; Sternad, D. Motor learning: Changes in the structure of variability in a redundant task. Adv. Exp. Med. Biol. 2009, 629, 439-456. [CrossRef] [PubMed]

29. Skurvydas, A.; Zachovajevas, P. Is post-tetanic potentiation, low frequency fatigue (LFF) and pre-contractile depression (PCD) coexistent in intermittent isometric exercises of maximal intensity? Acta Physiol. Scand. Suppl. 1998, 164, 127-133. [CrossRef] [PubMed]

30. Seitz, L.B.; Haff, G.G. Factors modulating post-activation potentiation of jump, sprint, throw, and upper-body ballistic performances: A systematic review with meta-analysis. Sports Med. 2016, 46, 231-240. [CrossRef] [PubMed]

31. Chaudhuri, A.; Behan, P.O. Fatigue in neurological disorders. Lancet 2014, 363, 978-988. [CrossRef]

(C) 2018 by the authors. Licensee MDPI, Basel, Switzerland. This article is an open access article distributed under the terms and conditions of the Creative Commons Attribution (CC BY) license (http:/ / creativecommons.org/licenses/by/4.0/). 